\title{
Políticas sociales, cuerpos y emociones a principios del siglo XIX en Argentina
}

Social policies, bodies and emotions in the early XIX century in Argentina

\author{
Rebeca Beatriz Cena / rebecena@hotmail.com \\ Universidad de Buenos Aires, Universidad de Mar del Plata, Consejo Nacional de \\ Investigaciones Cientificas y Técnicas, Argentina
}

\begin{abstract}
In this paper I will discuss - from an approach that recovers the sociology of the body and emotions - the first actions in the field of social policy in Argentina in the early nineteenth century, conducted by the Charitable Society of the Capital. From a qualitative analysis of secondary data, I will discuss the impact that government intervention possesses in terms of certain assumptions related to how the beneficiaries of social policies should behave. It is concluded that governmental interventions together with the regime of capitalist accumulation presuppose a set of emotions and sensitivities related to living in an abject deprivation condition.
\end{abstract}

Key words: Social policies, bodies/emotions policies, poverty, accumulation regime.

Resumen: En este trabajo se discutirá, desde un enfoque que recupera los aportes de la sociología del cuerpo y las emociones, las primeras acciones en materia de política social en Argentina a principios del siglo XIX, llevadas a cabo por la Sociedad de Beneficencia de la Capital. A partir de un análisis cualitativo de datos secundarios, se discute el impacto que la intervención estatal posee en términos de determinados supuestos vinculados al modo en que deben comportarse los actores destinatarios de la política social. Se concluye que las intervenciones gubernamentales, en sintonía con el régimen de acumulación capitalista, suponen un conjunto de emociones y sensibilidades vinculadas al hecho de vivir en situación de privación.

Palabras clave: políticas sociales, políticas de los cuerpos/emociones, pobreza, régimen de acumulación. 


\section{Introducción}

Las políticas sociales de atención a la pobreza —en tanto intervenciones estatales sobre aquellas poblaciones que se encuentran en condiciones de negación- constituyen un fenómeno a partir del cual analizar el régimen de acumulación imperante en una sociedad. Existe una vasta producción académica acerca del diseño, ejecución, evaluación e impacto de las políticas sociales; sin embargo, se encuentran pocos estudios que las problematicen en tanto políticas de los cuerpos y las emociones - es decir, en tanto estrategias que una sociedad implementa para dar respuesta a la disponibilidad social de los cuerpos (Scribano, 2009)-.

Las políticas sociales hacen sociedad (Danani, 1996) al implicar en sí mismas diversas maneras de intervención sobre el mundo social: qué problemáticas serán consideradas sociales, bajo qué criterios, qué sujetos quedarán bajo su cobertura, y, derivado de los anteriores, los impactos — quizás no del todo identificados y analizados por las ciencias sociales- sobre la conformación de las sensibilidad de las y los actores.

En este sentido, los regímenes de acumulación capitalista no sólo requieren regular los modos de producción, distribución y consumo de las mercancías, sino también regular los modos en que los sujetos se comportan (Turner, 1989). Aquellos que se encuentran imposibilitados de garantizar su reproducción autónomamente constituyen un nodo central de atención del capital, al menos, para regular y disminuir las potencialidades de conflictividad social asociadas a un número creciente de población viviendo en condiciones de negación (Offe, 1990).

El análisis de las políticas sociales desde una perspectiva sustentada en las políticas de los cuerpos/emociones constituye un nodo central de análisis de los modos en que el régimen de acumulación logra garantizar su persistencia en el tiempo pese a las situaciones de pobreza, desigualdad, exclusión y expulsión social que genera.

Todo régimen de acumulación posee un particular modo de regulación social y político que vuelva los comportamientos individuales compatibles con los requerimientos del régimen (Harvey, 2004). El modo de regulación, a partir de una de sus instituciones centrales - las políticas sociales de atención a la pobreza - se ancla no solamente en los comportamientos de los sujetos, sino previamente en las formas en que éstos perciben, comprenden, experimentan y sienten el mundo que los rodea. Así, las políticas sociales, de atención a la pobreza, constituyen una puerta de entrada para indagar cuál es 
la política de sensibilidad que el régimen de acumulación reserva para aquellos sectores que — necesariamente- son expulsados por los requerimientos del régimen.

El objetivo del presente radica en indagar las potencialidades analíticas de las categorías propuestas, a partir del análisis de los modos de intervención de la Sociedad de Beneficencia de la Capital ${ }^{1}$ en Argentina. En primer lugar, se realiza un abordaje de lo que se ha comprendido por política social y sus vinculaciones en términos del régimen de acumulación capitalista. En segundo lugar, se exponen las conexiones entre el primer apartado y las políticas de los cuerpos/emociones.

Se argumenta que las políticas sociales poseen un determinado régimen de sensibilidad que habilita/inhabilita determinados comportamientos emocionales. En tercer lugar, se analiza la Sociedad a partir del enfoque analítico propuesto dando cuenta del régimen de sensibilidad que suponían por parte de los sectores bajo su intervención. En cuarto lugar, se ofrece una serie de reflexiones finales que intentan recuperar las potencialidades del enfoque propuesto.

Como estrategia metodológica se ha trabajado a partir de datos secundarios (Scribano y De Sena, 2009) que otros investigadores han producido sobre la mencionada institución. "El análisis secundario implica la utilización de datos existentes, recogidos a los efectos de un estudio previo, con el fin de usarlos en una investigación de un interés que es distinto al de la indagación original, que podrá (involucrar) una nueva pregunta de investigación o una perspectiva alternativa sobre la pregunta original" (Heaton, 1998 citado en Scribano y De Sena, 2009: 103-104). Se trata, como han expuesto los autores, de hacer nuevas preguntas a viejos datos.

\section{Políticas sociales de atención a la pobreza}

Las políticas sociales como objeto de análisis, al menos desde Latinoamérica, se han vuelto un elemento ineludible para las ciencias sociales. Los persistentes índices de pobreza, desempleo y desigualdad social de la región (CEPAL, 2010, 2012, 2014) - y de todo el sur global- las han posicionado como un elemento esencial de análisis. Destacados autores se han centrado en dar cuenta de los impactos que las mismas poseen en los procesos de estructuración y estratificación social (Esping-Andersen, 1993; Adelantado, Noguera y Rambla, 2000; Martínez Franzoni, 2005; Rodríguez Enriquez, 2011; Valen-

1 De ahora en adelante la Sociedad o SBC. 
cia Lomelí, 2008; Villatoro, 2004). Dentro de la literatura se han constituido también en un instrumento de análisis para indagar los modos en que los diferentes regímenes de acumulación capitalista garantizan su persistencia a largo plazo, a través del manejo de los niveles de conflictividad social de las poblaciones en condiciones de pobreza (Offe, 1990; Grassi, 2003; Yazbek, 2000; Fleury, 2006).

Tal como propone Arriagada (2005), el modo sobre cómo la pobreza se define determina tanto las formas de medirla como las políticas para superarla. De la misma manera podríamos afirmar que el análisis de las políticas sociales destinadas a la pobreza nos permite decir algo acerca de las definiciones y modos de medirla que operan. En este sentido, Grassi (2003) ha sostenido que las políticas sociales establecen la nominación, definición y abordaje de lo que, en determinados momentos históricos, se posiciona como una problemática de carácter social. Ello se traduce en la capacidad gubernamental de imponer en escena pública un aspecto de la realidad definido como socialmente problemático, demandante de la intervención estatal y legítimamente reivindicable para las poblaciones reconocidas bajo la delimitación de dicha problemática (Cena, 2014).

Como otros autores se han encargado ya de argumentar (Adelantado et al., 2000), las políticas sociales en general, aunque las de atención a la pobreza en particular, afectan las relaciones de igualdad y desigualdad en una sociedad. Con ello refieren que las intervenciones gubernamentales pueden producir, disminuir o aumentar las desigualdades existentes. Expresión de ello lo constituyen, por ejemplo, las desigualdades en las cargas familiares que algunos titulares de las políticas sociales deben enfrentar, dado que, como veremos más adelante, este tipo de intervenciones sobre la pobreza ha vinculado el género con determinadas predisposiciones para la reproducción y producción de los hogares (Pautassi, 2009; Faret, 2011; Cena 2013).

Tal es el caso también del aumento de las desigualdades que se generan a partir de la exclusión de los sectores más vulnerables, producto de las restricciones y extrema focalización que muchas veces, desde el diseño mismo de la política social, no logran abarcar completamente al sector poblacional en condiciones de negación (Sojo, 2007).

Sumado a ello, estas formas de intervención gubernamental sobre las poblaciones ocupan un lugar nodal en los regímenes de acumulación capitalista (Harvey, 2004). En el clásico escrito de Offe (1990) sobre las políticas sociales, se argumenta el lugar ambiguo y contradictorio que ocupan, dado que materializan una respuesta gubernamental a corto plazo para satisfacer 
las "reivindicaciones populares", como una estrategia de largo plazo para el sostenimiento de la acumulación.

En otras palabras, toman en cuenta como factores causales tanto las exigencias de las clases trabajadoras como los requisitos sistémicos del capital, de allí que nunca puedan hacerle frente a ambos de forma coherente. Tienen la función de compensar la sociedad delimitando la definición de los temas, tiempos y métodos del conflicto, y así el establecimiento del marco - y no el resultado- del mismo. A la vez que expresan la mediana resolución de los intereses encontrados al tener la capacidad política de transmitir visiones del mundo acerca de la pobreza, sus causas, los responsables/responsabilizados, lo que es normal y lo no normal, etcétera. ${ }^{2}$

En este sentido, al ser parte del régimen de acumulación —-mediante el modo de regulación social y político (Harvey, 2004) — son parte de procesos que generan desigualdades, exclusiones y expulsiones sociales, estableciendo, al mismo tiempo, mecanismos determinados que ponen límites a esos procesos. De aquí deriva que las políticas sociales son elementos claves a partir de los cuales los regímenes de acumulación pueden garantizar determinados comportamientos de los sujetos que sean compatibles con su reproducción. Ello no solamente porque permiten disminuir los niveles de conflictividad social (Offe, 1990), como hemos adelantado previamente, sino porque además son un aspecto central del modo de regulación social y político (Neffa, 2006).

\section{Políticas de los cuerpos/emociones}

Las políticas sociales afectan los modos en que la sociedad se produce y reproduce al intervenir directamente sobre las formas en que se define una determinada situación como problema, los supuestos y redes conceptuales desde donde éstas han sido formuladas y diseñadas; la ejecución misma de la política social y una determinada gestión de las sensibilidades de los sujetos en condiciones de pobreza. Este último punto implica incorporar a su análisis una perspectiva que recupere los aportes de la sociología del cuerpo/ emociones. $^{3}$

2 "Si las políticas sociales desde el ángulo de los intereses del Estado asumen características funcionales al control social y a la propia reproducción de las condiciones de dominación, también presentan un carácter contradictorio que precisa ser considerado y que hace referencia a la intensidad de las luchas políticas de los subalternos por la ampliación de los programas y de las políticas de corte social" (Yazbek, 2000: 126).

3 La sociología se ha interesado, desde sus comienzos, por la condición corporal y emocio- 
Según Luna Zamora (2000), es posible reconocer en la sociología de las emociones la consolidación de un campo disciplinario a partir de mediados de 1970, aunque desde los clásicos de la sociología pueden rastrearse ya significativos antecedentes (Illouz, 2007; Scribano, 2013). En esa misma línea argumentativa, Kemper (1990) establece que en los años sesenta una serie de producciones desde la sociología imputan la lógica lineal y el interés por las estructuras sociales que han relegado al actor social y su vida emocional. "La sociología de las emociones exige entonces una mayor atención al 'yo' en relación con las estructuras sociales, sin desdibujar al actor social propiamente dicho, y subraya la necesidad de entender los fenómenos emocionales como fenómenos sociológicos" (McCarthy 1989: 63, citado en Luna Zamora, 2000: 3). Ello implicó el inicio de un camino dentro de la sociología que permitió la construcción de enlaces entre las dimensiones micro y macrosociológicas (Collins, 1990). En resumidas cuentas, este campo de la sociología comenzó a prestar atención a los factores sociales que influyen en la esfera corporal/emocional.

En esta línea, los regímenes de acumulación capitalista no sólo requieren regular los modos de producción, distribución y consumo de las mercancías, sino también regular los modos en que los sujetos se comportan (Turner, 1989). Aquellos que se encuentran imposibilitados de garantizar su reproducción autónomamente constituyen un nodo central de atención del capital, al menos, para regular y disminuir las potencialidades de conflictividad social asociadas a un número creciente de población viviendo en condiciones de pobreza. En este sentido, el análisis de las políticas sociales, desde una perspectiva sustentada en las políticas de los cuerpos/emociones, constituye un nodo central de análisis de los modos en que el régimen de acumulación logra garantizar su persistencia en el tiempo pese a las situaciones de pobreza que genera.

La organización y regulación de los cuerpos ha formado parte de los aspectos centrales de las sociedades desde el aislamiento de las conductas consideradas patológicas (Foucault, 2012), las desinfecciones de los hogares por parte de las administraciones públicas (Armus, 2000), hasta los modos que ha asumido y asume la sexualidad, el uso de los cuerpos y la procreación (Turner, 1989; Foucault, 2014; Beauvoir, 2014; Butler, 2012).

nal de los sujetos, aunque desde la segunda mitad del siglo XX se constituyen como campos disciplinares la sociología del cuerpo, por un lado, y la de las emociones por otro. Aquí sostendremos que ambos aspectos son inescindibles: no hay posibilidad de entender las acciones sociales si éstas no son realizadas desde y en la corporeidad, en su atravesamiento por componentes afectivos-emotivos. 
En este sentido, las políticas de los cuerpos, entendidas como la expresión que adquieren dentro del régimen de acumulación capitalista una serie de "estrategias que una sociedad acepta para dar respuesta a la disponibilidad social de los individuos en tanto parte de la estructuración social del poder" (Scribano, 2009: 1), atraviesan todas las instituciones de una sociedad. Sólo basta reflexionar acerca de los controles que, en términos de lo que es considerado como saludable, deben cumplir los trabajadores no solamente mientras se desarrollan en el ámbito laboral, sino también una serie de las denominadas "competencias emocionales" (Illouz, 2007), consideradas positivas para el desempeño dentro del ámbito laboral (Lisdero y Quattrini, 2013); las aptitudes emocionales que transmiten, cultivan y premian las escuelas en nuestras sociedades, vinculadas a un particular estilo de socialización mediada por reglas y roles de regulación y ordenamiento de los cuerpos; las vacunaciones masivas a las poblaciones contra las epidemias, que muchas veces adquirieron un carácter compulsivo y con presencia policial, particularmente en el caso de los sectores mayormente empobrecidos (Armus, 2000).

Las políticas de los cuerpos implican también una determinada política de las emociones ${ }^{4}$ que regulen los modos en que los actores experimentan los "estados de sentirse y sentir el mundo [y vehiculicen] las percepciones asociadas a las formas socialmente construidas de las sensaciones" (Cervio, 2012: 11). Las mismas se encuentran acompañadas por los dispositivos de regulación de las sensaciones y mecanismos de soportabilidad social (Scribano, 2010), como las formas socialmente existentes de ordenar, categorizar y clasificar las emociones, configurando las sensibilidades sociales, en el caso de los primeros; y en los segundos, evitar sistemáticamente el conflicto social. Ambos traman y constituyen el régimen de sensibilidad que una sociedad construye para sus propios integrantes.

En sintonía con lo que propone McCarthy (1989), las emociones son comprendidas aquí como procesos sociales, es decir, socialmente construidas. ${ }^{5}$ De allí que no pueden ser abordadas como estados internos de los su-

4 "Las emociones se enraízan en los estados del sentir el mundo que permiten vehiculizar las percepciones asociadas a formas socialmente construidas de sensaciones. Los sentidos orgánicos y sociales permiten vehiculizar aquello que parece único e irrepetible como son las sensaciones individuales, y elaboran a la vez el 'trabajo desapercibido' de la incorporación de lo social hecho emoción” (Scribano, 2007: 122).

5 'Las 'situaciones emocionales' se inscriben dentro de modelos relativamente sostenidos y perdurables de relaciones sociales. En este orden, aún las llamadas emociones primarias y universales están también sujetas a estos condicionamientos de la estructura social, conside- 
jetos, individuales y aislados. En otras palabras, constituyen acciones directamente dirigidas a y causadas por la interacción con otros en un contexto y situación social (Matthews, 1992).

Los cuerpos constituyen más que meros instrumentos de mediación de las emociones, las maneras de experimentar, pertenecer y ser en el mundo. Ello implica, entre otros aspectos, comprender que las y los actores se comportan desde y a través de su materialidad corpórea, y en ese sentido, no hay acción social posible sin cuerpo. Los cuerpos ocupan así un lugar central como locus de la conflictividad y el orden.

Los sistemas capitalistas de la actualidad, como maneras de estructuración de las sociedades, con particularidades en las latinoamericanas, requieren como sitios privilegiados el control de los cuerpos, de manera tal de delimitar, posibilitar y propiciar determinadas maneras de actuar/sentir de las y los actores.

El análisis de las políticas sociales en tanto parte constitutiva de una particular política de/sobre los cuerpos/emociones se vuelve ineludible dado que la materialidad corpórea ocupa un lugar central en la producción y clasificación de las sensaciones, comenzando por los sentidos. "[E]l ojo que ve, la boca que degusta, la nariz que huele, el oído que escucha y la piel que toca, son terminales sensoriales tan físico-biológicas como histórico-sociales a partir de las cuales el sujeto entabla relaciones y configura las maneras del sentir(se) en/con las cosas, con los otros y con sí mismo" (Cervio, 2012: 10).

Debido a su carácter histórico-social, las sociedades producen y reproducen particulares maneras de regular, ordenar y "hacer cuerpo" determinadas prácticas en términos de un particular régimen de sensibilidad. Dicho régimen de sensibilidad regula y vuelve soportable las formas en que se perciben los sentires sobre el mundo, siendo ambos mecanismos (regular y volver soportable) condición de posibilidad de la reproducción del capitalismo a largo plazo. El modo de regulación social y político al que anteriormente referíamos se ancla no solamente en los comportamientos de los sujetos, sino previamente, en las formas en que éstos perciben, comprenden, experimentan y sienten el mundo que los rodea. Así, las políticas sociales, particularmente las de atención a la pobreza, constituyen una puerta de entrada para indagar cuál es la política de sensibilidad que el régimen de acumulación reserva para aquellos sectores que, necesariamente, son expulsados por los requerimientos del régimen.

ración en la cual coincido" (Luna Zamora, 2000: 5). 
La constitución de los cuerpos/emociones, cómo las y los actores se vinculan y experiencian el mundo, si bien pueden ser analizadas como respuestas neurofisiológicas heredadas, se encuentran vinculadas a modelos y esquemas sociales. Todo lo que las emociones poseen en tanto "sentimiento" experimentado por la subjetividad de las y los actores requieren de explicaciones científicas que las anclen al orden social en el cual la/el actor se desempeña. "Es decir, los sentimientos y emociones subjetivas además de tener una intercontextualidad -situación-, tienen que ver con un contexto histórico cultural determinado, que es el que le ofrece al individuo sus códigos para sentir y expresar sus vivencias emocionales y afectivas de manera efectiva" (Luna Zamora, 2007: 1).

Las emociones, desde esta perspectiva, no tienen tanto que ver con procesos individuales e individualizables - aunque sean narrados en primera persona como lo más íntimo y propio que le sucede a los sujetos-, sino que constituyen relaciones sociales resultado de constructos históricos, sociales y culturales expresadas a través de una trama de símbolos a partir de los cuales los sujetos pueden inferir significados. El modo sobre cómo los actores experimentan el mundo que los rodea, si bien posee algo de individual y subjetivo - en tanto terminales sensoriales de una corporalidad - posee al mismo tiempo un lenguaje susceptible de ser reconocido por los pares (Le Breton, 2012).

En otras palabras, los análisis realizados desde la sociología de los cuerpos/emociones implica no prestar atención a aquello entendido como estrictamente "subjetivo", sino que dando un paso más adelante comprende que cada una de las experiencias emocionales responden a determinados procesos de estructuración social que las significan y dan sentido (Shweder, 1994). "De esta suerte, se interesa por comprender hasta qué punto sentir determinadas emociones, y expresarlas de un modo y no de otro está estrechamente ligado a la clase social a la que se pertenece, al lenguaje y los referentes aprendidos, a las nociones de qué es lo 'propio’ o más adecuado a cada situación, a cada género y grupo de edad” (Luna Zamora, 2000: 4).

Un abordaje sociológico de los cuerpos/emociones implica comprender que aquello que pareciera posicionarse y experimentarse en la esfera subjetiva de los actores, como son las emociones, poseen un fuerte correlato con normas sociales, costumbres, tradiciones institucionales - como las de las políticas sociales-, que en tanto procesos estructurales no solamente las significan, sino también las posibilitan, de allí que determinados contextos sociales propicien determinados comportamientos emocionales y restrinjan otros (Gordon, 1990; Hochschild, 1990). 


\section{El régimen de sensibilidad impartido hacia los sectores empobrecidos a comienzos del siglo XIX}

La política social en Argentina no se desarrolla, tal y como la hemos conceptualizado en este trabajo, sino hasta principios del siglo XIX cuando comienzan las primeras preocupaciones por la cuestión social con la conocida Sociedad de Beneficencia de la Capital (y sus modalidades en el Interior). Esta institución con participación estatal data en Argentina de 1823 cuando el presidente Bernardino Rivadavia, a través del decreto del 2 de enero de ese mismo año, le otorga existencia. Fue constituida como una institución que disponía de recursos en gran medida estatales y diversas donaciones de particulares, administrada privadamente por un grupo de mujeres de la clase alta porteña. La peculiaridad de esta actividad vinculada a la asistencia social, respecto a las otras funciones centralizadas por el Estado, radicaba en que actuaba por delegación, al encarar el Estado las problemáticas sociales indirectamente a través de la provisión de fondos ${ }^{6}$ hacia una institución de gestión privada.

La particularidad de esta institución con fondos públicos, pero de gestión privada, produjo constantes tensiones a lo largo de su existencia. Por un lado, en disputa con la Iglesia ${ }^{7}$ que venía siendo desplazada por el carácter laicista del Estado liberal y, por otro, con el Estado, dado que al tiempo que la institución reclamaba la atención de éste, sobre todo financiera, propugnaban por la libertad en las decisiones respecto a formas de organización y funcionamiento de las instituciones que centralizaban (Guy, 2000).

Los principales destinatarios de las acciones de esta organización fueron las mujeres, ancianos y niños empobrecidos. Las intervenciones que llevaba adelante la Sociedad buscaban modificar comportamientos, aptitudes y ca-

6 Si bien la Sociedad recibía algunos aportes y donaciones de privados, éstos no representaban grandes partidas "algunas donaciones se hacían en vida y la mayoría de las veces no eran abultadas, y en el conjunto de los recursos disponibles por la Sociedad las donaciones no significaban más del 10 o 15 por ciento de todos ellos" (Moreno, 2000: 13). La mayoría de sus ingresos provenían de las transferencias estatales que desde 1893 le concedía un porcentaje de las Loterías (Moreno, 2000).

7 Estos conflictos institucionales, primero del Estado Español y, luego de la independencia, del Estado Argentino, con la Iglesia tuvieron sus primeras expresiones a finales del siglo XVIII (Moreno, 2000). Con la creación de la Sociedad en 1823, se reemplaza a la Hermandad [de la Santa Caridad] en el manejo de las instituciones relacionadas con los cuidados sanitarios -como hospitales-y casas de huérfanos. 
pacidades de los destinatarios so pretexto de modificar las condiciones de pobreza y miseria en las que vivían. Un fuerte rasgo de la institución, y de la época, fue que, más allá de su labor en la fundación de algunas escuelas y hospitales de la capital (Moreno, 2000), su participación en la vida de las poblaciones vulneradas tenía más un rasgo moralizador. "[L]a población sobre la cual actúa 'la Beneficencia', empieza a estar definida por la asociación mecánica entre inmoralidad y miseria en un mundo en donde la moralidad y el trabajo son vistos como instrumentos de movilidad social ascendente" (Soldano y Andrenacci, 2006: 58). Se suponía que modificando los comportamientos de las poblaciones podrían modificarse las situaciones de carencias en las que vivían, como si dichas condiciones de vida se debieran a aspectos particulares e individualizables en los sujetos.

Su carácter de gestión privada le otorgaba a la institución cierta autonomía en las decisiones. ${ }^{8}$ Las mujeres de la oligarquía que la administraban, intentaban fundar valores como la humildad, el valor de la mujer argentina pobre y la pobreza virtuosa. En esta institución, que comenzó a esbozar los primeros pasos de la política social en Argentina, se explicitan determinadas políticas de los cuerpos/emociones vinculadas a los comportamientos que la Sociedad creía eran los idóneos por parte de los sectores empobrecidos. Esto puede verse traducido en determinadas competencias emocionales (Illouz, 2007) que debían poseer las personas en condiciones de pobreza, como la humildad y el amor filial y el cultivo del "buen sexo".

Tal como Grassi (1989) recupera en su escrito, en el discurso de creación de la Sociedad, el presidente de entonces sostenía que:

las mujeres de los sectores populares, [debían] producir "ellas mismas lo que no dan ahora" y adquirir "por sí mismas sus medios de subsistencia". Faltaban hombres para producir, pero también para garantizar la manutención de su familia. Para eso era necesario "instruir al bello sexo" permitirle "cultivar su razón y adornar su inteligencia". No obstante, el señor Ministro "creyó deber recomendar al bello sexo la circunspección y tino con que debía manejarse al emprender la carrera del cultivo de su espíritu. Al principio (dijo) todo debe ser sobrio y modesto para que llegue a ser sólido y eficaz [...] Por entonces, la moral de los pobres — para los ricos- pasaba por dos variables complementarias: la resignación y la obediencia, expresada en la observancia de aquellos principios que las clases dominantes estimaban adecuados para los pobres" (Grassi, 1989: 53-65).

8 Aunque dicha autonomía fue constantemente disputada por un Estado que comenzaba a concentrar y a centralizar las acciones destinadas a atender a niños, mujeres y enfermos (Guy, 2000). 
El manejo del cuerpo y las emociones de los considerados sectores pobres, a través de una institución que concentraba buena parte de los recursos destinados a las políticas sociales del momento, se hace evidente en el discurso de creación de ésta. Condición de posibilidad de superación de las situaciones de carencias eran determinadas competencias emocionales y aptitudes corporales vinculadas con la resignación, obediencia e inteligencia y a lo que en aquel entonces era considerado "el bello sexo".

Junto con esta serie de competencias emocionales, se impartía una serie de enseñanzas que buscaban fortalecer, principalmente en las mujeres pobres destinatarias de esta institución, el vínculo entre feminidad y maternidad, como elementos clave de la reproducción de la sociedad. La disciplina, impuesta a través de las intervenciones de la institución, buscaba garantizar la producción, reproducción y mejoramiento de la fuerza de trabajo urbana.

La asistencia social sigue teniendo el carácter de recurso del que el Estado debe disponer para sanear el cuerpo social, cuyo motivo esencial es una pedagogía disciplinatoria de los sectores populares, ideada por hombres y ejecutada por mujeres sobre mujeres, especialmente sobre el binomio madre-hijo. Pero ese carácter coexiste conflictivamente con la preocupación de "administrar una población" constituida como "capital humano", reorganizando las intervenciones de manera de garantizar la reproducción de la fuerza de trabajo urbana (Soldano y Andrenacci, 2006: 29).

En este caso, el binomio mujer-madre pasa casi desapercibidamente como algo dado, inalterable. La buena madre se posiciona como una condición no solamente deseable, sino también incentivada desde estos espacios de intervención sobre los sectores empobrecidos.

El diagnóstico de partida consideraba que la miseria, indigencia y pobreza eran producto de debilidades morales de los actores que la padecían. Al mismo tiempo, esta forma de intervención se encontraba amparada en la idea de peligrosidad que pueden generar los pobres en cuanto a desarmonizar el orden social. De allí su preocupación por fijar adecuadamente los destinatarios de la intervención como los niños huérfanos, madres abandonadas o solteras, las personas consideradas pobres y delincuentes:

En consecuencia, la política asistencial tenderá a ser cada vez más productiva y orientada a la producción de individuos sanos, fuertes y disciplinados, esto es: aptos para el trabajo asalariado regular; 9 en este sentido la población-riqueza, la población-mano de obra o capacidad de trabajo, la población en equilibrio entre su propio crecimiento y los recursos de que dispone será el objeto y objetivo de la asistencia social pública (Fanfani, 1989: 619, citado en Rozas Pagaza, 2004: 77).

9 Las cursivas son propias. 
Estas formas de intervención concebían la cuestión social como una problemática meramente individual y, por ende, al actor como generador de sus propios problemas y soluciones - esto es, con los suficientes instrumentos como para revertir las condiciones de carencias-. De allí que buscara modificar constantemente aquello que, desde la sociología de los cuerpos/emociones, se ha denominado como competencias emocionales que inclinaran a los sujetos hacia la disciplina y el uso de su fuerza corporal que los volvieran "aptos" para el mercado laboral.

Las acciones de esta institución porteña estaban guiadas por la filantropía y la tutela. Esto es, se comprendía que existían seres humanos que no podían hacer frente a la satisfacción de sus necesidades por imposibilidades propias - ya sean físicas o morales - de allí que necesitaran la acción solidaria de quienes sí pudieron, se entiende, a través del esfuerzo y la perseverancia. Desde esta institución el motivo esencial era una pedagogía disciplinadora de los sectores carenciados.

También se entendía que quienes no podían satisfacer sus necesidades carecían, al mismo tiempo, de los valores morales necesarios para revertir su situación. De allí la necesidad de "alguien" — considerado superior- que direccione sus conductas. Esta orientación moralista y naturalizante de la cuestión social identificable en el accionar de la institución a lo largo de su existencia da cuenta de una concepción que pasaba más por aspectos religiosos, morales y de disciplinamiento que por cuestiones político-económicas.

Se consideraba que la principal razón de las carencias de las personas eran individuales, esto es, que las condiciones de precariedad e insatisfacción de las necesidades sociales era producto de conductas, hábitos y debilidad moral de quienes la padecían. Las iniciativas de esta organización poseían un fuerte sesgo moralizante y la clasificación de los pobres respondía a una estrategia para mantener el orden e integración en la sociedad. Consideraban que se era pobre por azar (como la enfermedad, la muerte o el accidente) o por inmoralidad.

De allí que hayan puesto en juego dos estrategias significativas. Por un lado, la "sanción ejemplificadora", que consistía en el llamado premio a la virtud. Dentro de las prácticas de la institución, una vez al año la Sociedad realizaba un acto en el Teatro Colón, ${ }^{10}$ donde se otorgaban premios a las mujeres

10 El Teatro Colón se encuentra ubicado a pocos metros del sector más reconocido de la Ciudad Autónoma de Buenos Aires (capital de Argentina): el monumento llamado Obelisco. Representa el principal centro de producción, reproducción y difusión de lo que concierne a las danzas clásicas, óperas, ballet, etcétera. 
que cumplían con ciertos requisitos morales. A esta ceremonia eran invitadas personalidades públicas como el presidente de la nación, ministros, arzobispos, entre otras figuras.

Los "premios a la virtud" que la Sociedad de Beneficencia otorgaba desde 1823, informan con singular consistencia esta apreciación. Anualmente, en acto público celebrado en el Teatro Colón, la Comisión Directiva de la Sociedad de Beneficencia entregaba premios a asistidos considerados merecedores de los mismos, quienes "comparecían ante la mesa oficial" para que le sean entregados los diplomas respectivos (Grassi, 1989: 65).

Mediante este reconocimiento y exposición pública se difundían aquellos principios y valores que los centros de alto poder político y adquisitivo consideraban relevantes impartir a los sectores empobrecidos de la época. Ello no solamente significaba toda una ceremonia de premiación, sino de institucionalización de determinados valores y virtudes que, impuestos por un sector de la sociedad, eran reconocidos como "los valores" que debían cumplir los sectores en condiciones de pobreza.

Dentro de las "virtudes" premiadas se encontraban:

humildad; amor filial; desinterés; a la persona menesterosa que haya manifestado constancia en el trabajo; a la persona enferma más paciente; al amor conyugal; a la viuda con hijos, pobre y con virtudes cristianas; a la mujer argentina pobre que se distinga en el orden y arreglo de su hogar; a las familias vergonzantes; a las familias desgraciadas; a tres huérfanas con buena conducta, humildad y amor al trabajo; a la anciana que viva con el producto de su trabajo de costura; a la viuda pobre vergonzante; a la mujer más sufrida y pobre (Grassi, 1989: 65-66). Conjuntamente con ello, se obligaba "a los niños internos de los asilos y orfanatos dependientes de la SBC a pedir limosna 'uniformados' con las cabezas rapadas" (Golbert, 1996: 5).

Como puede observarse dentro de las competencias emocionales y corporales que la Sociedad consideraba significativas se encontraban aquellas que impartían la obediencia (a la persona enferma más paciente, la humildad), la resignación, la perseverancia en el trabajo (el amor y constancia en el trabajo, a la persona que viva del producto de su trabajo de costura) y aquellos principios considerados como propios del género femenino (el ser madre, el amor y cuidado de los hijos, que mantengan el orden y arreglo de su hogar).

\section{Reflexiones}

El enfoque de la sociología del cuerpo y las emociones abona el abordaje teórico propuesto, dado que las maneras en que los actores sienten, desean, gustan, oyen e interactúan se encuentran mediadas por las estructuras del ré- 
gimen que posibilitan o imposibilitan la expresión de alguno de ellos. Desde esta perspectiva, un análisis de la política de los cuerpos/emociones se vuelve ineludible para las ciencias sociales, pues implican un análisis de un "nicho" de dominación que se presenta como lo más íntimo y privado de los actores; esto es, cómo viven ellos, experimentan y sienten sus emociones. Ello adquiere aún más potencialidad analítica cuando estamos preguntándonos por las políticas de los cuerpos/emociones de un sector de la población particularmente afectado en la satisfacción de sus necesidades y potencialmente conflictivo para el capital.

Las políticas sociales permiten comprender algunas de las estrategias del régimen de acumulación que bajo el manto de la "ayuda social" o la "beneficencia” contribuyen a su reproducción a largo plazo, a su persistencia en el tiempo y a la disminución de los niveles de conflictividad social. El enfoque de las políticas de los cuerpos/emociones da un paso más adelante descifrando los modos de sentir, pensar, experimentar y actuar que resultan compatibles con los requerimientos del régimen y son complementarios de las políticas sociales. Desde este punto de vista, las políticas sociales requieren y suponen un tipo de comportamiento por parte de los destinatarios. Materializan, de esta manera, una serie de normas, costumbres, tradiciones y creencias institucionalizadas que promueven ciertos comportamientos emocionales y restringen otros.

De esta forma, el análisis de la Sociedad, como expresión metonímica del fenómeno en estudio, permite dar cuenta de los cuerpos/emociones en condiciones de pobreza compatibles con el régimen de acumulación. En su momento, la Sociedad de Beneficencia de la Capital no solamente pudo definir cuáles eran las problemáticas sociales de su tiempo, sino también cuáles eran las soluciones.

La mujer como "buena madre" y encargada de la reproducción del hogar es un supuesto que ha acompañado a la historia de las políticas sociales de atención a la pobreza ${ }^{11}$ : posicionándose en el actor privilegiado de la intervención estatal, con determinadas competencias emocionales que garantizan la reproducción de la esfera doméstica. Esta presentación cuasi naturalizada de la mujer como madre, ocluye y desconoce no solamente su no-naturalidad, sino también el papel indispensable que este rol asignado a la mujer en la esfera doméstica implica para el desempeño de las economías capitalistas actuales.

11 Véase al respecto Pautassi (2009). 
El discurso de la Sociedad, en tanto discurso dominante, deposita en aptitudes emocionales de los sujetos la causa última de las condiciones de pobreza que padecen, y en la modificación de las mismas las posibilidades de superación. Este efecto culpabilizador alimenta, consolida y reproduce las distancias sociales: un otro poderoso, que maneja recursos económicos y simbólicos contribuye a definir la situación problema en tanto cuestión individual oscureciendo la naturaleza política y económica de tales condiciones de negación, abriendo camino así a la tolerancia social.

A partir de la distribución de bienes y servicios y reconocimientos, quienes pertenecen a los sectores más desfavorecidos han convivido y se han adaptado a un discurso dominante que desdibuja los procesos de estructuración social que desembocaron en las situaciones de carencias. En resumidas cuentas, las políticas sociales, en tanto parte de las políticas de los cuerpos/ emociones del régimen de acumulación, forman parte de las dimensiones simbólicas y relacionales que contribuyen a crear, mantener y reproducir la privación.

\section{Bibliografía}

Adelantado, José, José Noguera y Xavier Rambla (2000), "El Marco de Análisis: las relaciones complejas entre estructura social y políticas sociales", en Adelantado, José [comp.], Cambios en el Estado de Bienestar. Politicas Sociales y Desigualdades en España, Barcelona: Icaria.

Arriagada, Irma (2005), "Dimensiones de la pobreza y políticas desde una perspectiva de género", en Revista de la CEPAL, núm. 85, Santiago de Chile: CEPAL.

Armus, Diego (2000), "El descubrimiento de la enfermedad como problema social", en Mirta Lobato [dir.], El progreso, la modernización y sus límites (1880-1916), Nueva historia argentina, vol. 5, Buenos Aires: Sudamericana.

Beauvoir, Simone de (2014), El segundo sexo, Buenos Aires: Debolsillo.

Butler, Judith (2012), Cuerpos que importan: sobre los limites materiales y discursivos del "sexo", Buenos Aires: Paidós.

Cena, Rebeca (2014), "Imagen Mundo y Régimen de sensibilidad. Un análisis a partir de las políticas sociales de atención a la pobreza implementadas en Argentina", en Revista Latinoamericana de Estudios sobre Cuerpos, Emociones y Sociedad, Córdoba: CIECS.

Cena, Rebeca (2014), "Programas de transferencias condicionadas de ingresos y programas de empleo en Argentina: entre la responsabilización de los destinatarios y la individualización de la cuestión social", en Boletín Cientifico Sapiens Research, vol. 4, núm. 1, Colombia.

Cervio, Ana (2012), Tramas del Sentir: ensayos desde una sociología de los cuerpos y las emociones, Buenos Aires: Estudios Sociológicos Editora. 
Rebeca Beatriz Cena. Politicas sociales, cuerpos y emociones a principios del siglo XIX en Argentina

Collins, Randall (1990), “Stratification, Emotional Energy, and the Transient Emotions”, en Theodore Kemper [ed.], Research Agenda in the Sociology of Emotions, New York: State University of New York Press.

Danani, Claudia (1996), "Algunas precisiones sobre la política social como campo de estudio y la noción de población objeto", en Hintze, Susana [coord.], Políticas sociales: contribución al debate teórico-metodológico, Buenos Aires: CEA/UBA.

Esping-Andersen, Gosta (1993), Los tres mundos del Estado de Bienestar, Valencia: Edicions Alfons El Magnanim.

Faret, Pablo (2011), Documento de Trabajo núm. 2: Antecedentes para el análisis de los Programas de Transferencias Condicionadas de América Latina desde un enfoque de Derechos: El Principio de Igualdad y No Discriminación, Santiago de Chile: FAO, MIMEO.

Fleury, Sonia (2006), Democracia, ciudadanía y políticas sociales, vol. 2, núm. 2, Buenos Aires: Universidad Nacional de Lanús.

Foucault, Michel (2012), Vigilar y castigar: el nacimiento de la prisión, Buenos Aires: Siglo XXI.

Foucault, Michel (2014), Historia de la Sexualidad, Buenos Aires: Siglo XXI.

Grassi, Estela (1989), Las mujeres y la profesión de asistente social. El control de la vida cotidiana, Buenos Aires: Humanitas.

Grassi, Estela (2003), "Políticas de Asistencia Focalizadas en el Desempleo y la Pobreza”, en Grassi, Estela, Politicas y problemas sociales en la sociedad neoliberal. La otra década infame (I), Buenos Aires: Espacio Editorial.

Gordon, Steven (1990), “Social Structural Effects on Emotions", en Kemper, Theodore [ed.], Research Agenda in the Sociology of Emotions, New York: State University of New York Press.

Guy, Donna (2000), “La 'verdadera historia' de la Sociedad de Beneficencia”, en Moreno, José Luis, La politica social antes de la política social: caridad, beneficencia y política social en Buenos Aires. Siglos XVIII a XX, Buenos Aires: Prometeo-Trama.

Harvey, David (2004), “Introducción”, en La Condición de la Posmodernidad. Investigación sobre los orígenes del cambio cultural, Buenos Aires: Amorrortu.

Hochschild, Arlie (1990), "Ideology and Emotion Management: A Perspective and Path for Future Research", en Kemper, Theodore [ed.], Research Agenda in the Sociology of Emotions, New York: State University of Nueva York Press.

Illouz, Eva (2007), Intimidades Congeladas. Las emociones en el capitalismo, Buenos Aires: Katz Editores.

Kemper, Theodore (1990), “Themes and Variations in the Sociology of Emotions", en Kemper, Theodore [ed.], Research Agenda in the Sociology of Emotions, New York: State University of New York Press.

Luna Zamora, Rogelio (2007), "Emociones y subjetividades. Continuidades y discontinuidades en los modelos culturales", en Luna Zamora, Rogelio y Adrián Scribano [comp.], Contigo Aprendi... Estudios Sociales de las Emociones, Córdoba: CEA, CONICET, Universidad Nacional de Córdoba, CUSCH, Universidad de Guadalajara. 
Luna Zamora, Rogelio (2000), “Introducción a la Sociología de las Emociones”, en Revista Universidad de Guadalajara, primavera, año 18, Guadalajara: UDG.

Martínez Franzoni, Juliana (2005), "Regímenes de bienestar en América Latina: consideraciones generales e itinerarios regionales", en Revista Centroamericana de Ciencias Sociales de FLACSO, vol. 4, núm. 2, Costa Rica: FLACSO.

Mathews, Holly (1992), “The Directive Force of Morality Tales in a Mexican Community”, en D'Andrade, Roy y Claudia Strauss, Human Motives and Cultural Models, Nueva York: Cambridge University Press.

McCarthy, Doyle (1989), "Emotions are Social Things: An Essay in the Sociology of Emotions", en Franks, David y Doyle McCarthy [eds.], The Sociology of Emotions: Original Essays and Research Papers, Greenwich, Connecticut, Londres: Jai Press Inc.

Moreno, José Luis (2000), La politica social antes de la politica social: caridad, beneficencia y politica social en Buenos Aires. Siglos XVIII a XX, Buenos Aires: Prometeo-Trama.

Offe, Claus (1990), Contradicciones en el Estado de bienestar, México: Alianza.

Pautassi, Laura (2009), "Programas de transferencias condicionadas de ingresos. ¿Quién pensó en el cuidado? La experiencia en Argentina”, en Seminario Regional Las familias latinoamericanas interrogadas. Hacia la articulación del diagnóstico, la legislación y las políticas, Santiago de Chile: CEPAL.

Rodríguez Enríquez, Corina (2011), Programas de transferencias condicionadas de ingreso e igualdad de género. ¿Por dónde anda América Latina?, Santiago de Chile: CEPAL.

Rozas Pagaza, Margarita (2004), La intervención profesional en relación con la cuestión social. El caso del trabajo social, Buenos Aires: Espacio Editorial.

Shweder, Richard (1994), “The Basics of Basic Emotion”, en Ekman, Paul y Richard Davidson [eds.], The Nature of Emotion. Fundamental questions, Nueva York: Oxford University Press.

Scribano, Adrián (2007), Vete tristeza... Viene con pereza y no me deja pensar!... Hacia una sociología del sentimiento de impotencia, Córdoba: CEA, UNC, CUSCH, UDEG.

Scribano, Adrián (2009), "La Sociedad hecha callo: conflictividad, dolor social y regulación de las sensaciones”, en Scribano, Adrián [comp.], Mapeando interiores. Cuerpo, conflicto y sensaciones, Córdoba: CEA, UNC, Jorge Sarmiento Editor.

Scribano, Adrián (2010), "Estados Represivos: Políticas de los Cuerpos y Prácticas del Sentir", en Revista Brasileira de Sociologia da Emoção, año 9, núm. 25, Brasil: Universidade Federal Da Paraíba.

Scribano, Adrián (2013), Teoría social, cuerpos y emociones, Buenos Aires: Estudios Sociológicos Editora.

Scribano, Adrián y Angélica De Sena (2009), “Las segundas partes sí pueden ser mejores: algunas reflexiones sobre el uso de datos secundarios en la investigación cualitativa”, en Sociologías, año 11, núm. 22, jul./dic., Porto Alegre.

Sojo, Ana (2007), "La trayectoria del vínculo entre políticas selectivas contra la pobreza y políticas sectoriales", en Revista CEPAL 91, Santiago de Chile: CEPAL.

Soldano, Daniela y Luciano Andrenacci (2006), “Aproximaciones a las teorías de la política social a partir del caso argentino", en Andrenacci, Luciano [comp.], Problemas de politica social en la Argentina contemporánea, Buenos Aires: UNGS/Prometeo. 
Rebeca Beatriz Cena. Politicas sociales, cuerpos y emociones a principios del siglo XIX en Argentina

Turner, Brian (1989), El cuerpo y la sociedad. Exploraciones en teoría social, México: Fondo de Cultura Económica.

Valencia Lomelí, Enrique (2008), "Las transferencias monetarias condicionadas como política social en América Latina. Un balance: aportes límites y debates”, en The Annual Review of Sociology, vol. 34, Canadá: Annual Reviews.

Villatoro, Pablo (2004), Programas de reducción de la pobreza en América Latina. Un análisis de cinco experiencias, Santiago de Chile: División de Desarrollo Social de la Comisión Económica para América Latina y el Caribe (CEPAL).

Yazbek, María Carmelita (2000), "Políticas Sociales y Asistenciales: Estrategias Contradictorias de Gestión Estatal de la Pobreza de las Clases Subalternas”, en Borgianni, Elisabete y Carlos Montaño [orgs.], La politica social hoy, San Pablo: Cortez Editora.

\section{Recursos electrónicos}

Comisión Económica para América Latina y el Caribe (2010). "Panorama Social de América Latina”. Disponible en: http://www.eclac.cl/cgi-bin/getprod.asp?xml=/ publicaciones $/ \mathrm{xml} / 9 / 41799 / \mathrm{P} 41799 . x m l \& x s l=/ \mathrm{tpl} / \mathrm{p} 9 f . x s l \& b a s e=/$ publicaciones $/$ top_publicaciones.xsl [25 de junio de 2012].

Comisión Económica para América Latina y el Caribe (2012), "Panorama Social de América Latina”. Disponible en: http://repositorio.cepal.org/bitstream/11362/1247/1/ S2012959_es.pdf [3 de agosto de 2013].

Comisión Económica para América Latina y el Caribe (2014), "Panorama Social de América Latina”. Disponible en: http://repositorio.cepal.org/bitstream/11362/37626/4/ S1420729_es.pdf [15 de febrero de 2015].

Golbert, Laura (1996), "Viejos y Nuevos Problemas de las Políticas Asistenciales”, en Serie de Estudios, núm. 12, Buenos Aires: Centro de Estudios para el Cambio Estructural. Disponible en: http://www.cece.org.ar/fse12.htm [15 de marzo de 2012].

Le Breton, David (2012), "Por una antropología de las emociones", en Revista Latinoamericana de Estudios sobre Cuerpos, Emociones y Sociedad, núm. 10, año 4, diciembre 2012-marzo de 2013, Argentina. Disponible en: http://www.relaces.com. ar/index.php/relaces/article/view/208 [11 de marzo de 2013].

Lisdero, Pedro y Diego Quattrini (2013), “Educación, corporalidad y nueva morfología del trabajo; Los calls centers ¿escuelas de trabajo?”, en Intersticios: Revista Sociológica de Pensamiento Critico, vol. 7, núm. 1. Disponible en: www.intersticios.es/article/ download/10572/7775 [11 de enero de 2014].

Neffa, José (2006), “Evolución Conceptual de la Teoría de la Regulación”, en, De La Garza Toledo, Enrique [comp.], Teorías Sociales y Estudios del Trabajo: Nuevos Enfoques, México: Anthropos. Disponible en: http://docencia.izt.uam.mx/egt/publicaciones/ libros/teoria_social/cap9.pdf [13 de noviembre de 2012].

Scribano, Adrián (2012), “Sociología de los cuerpos/emociones”, en Revista Latinoamericana de Estudios sobre Cuerpos, Emociones y Sociedad (RELACES), núm. 10, año 4, diciembre 2012-marzo de 2013, Córdoba. Disponible en: http://www.relaces.com. ar/index.php/relaces/article/view/224 [23 de junio de 2013]. 
Rebeca Beatriz Cena. Becaria doctoral del Consejo Nacional de Investigaciones Científicas y Técnicas. Docente de la carrera de sociología en la Universidad de Buenos Aires y en la Universidad de Mar del Plata. Miembro del Centro de Investigaciones y Estudios Sociológicos. Candidata a obtener el Doctorado en Ciencias Sociales de la Universidad de Buenos Aires, Argentina. Líneas de investigación: sociología del cuerpo/emociones vinculado con el análisis de las políticas sociales: cuerpos/emociones y régimen de acumulación; políticas sociales y régimen de regulación; cuerpos/emociones y procesos de estructuración social; políticas sociales y estructuración de las sensibilidades. Publicaciones recientes: "Imagen Mundo y Régimen de sensibilidad. Un análisis a partir de las políticas sociales de atención a la pobreza implementadas en Argentina", en Revista Latinoamericana de Estudios sobre Cuerpos, Emociones y Sociedad, núm. 14, año 6, abril-julio (2014a); "Fronteras complejas: protección social e inmigración. Asignación universal por hijo para protección social e inmigración en Argentina", en Revista Latinoamericana POLIS, núm. 38, agosto. Disponible en: http://polis.revues. org/10189;DOI: 10.4000/polis.10189 (2014b); "Programas de transferencias condicionadas de ingresos y programas de empleo en Argentina: entre la responsabilización de los destinatarios y la individualización de la cuestión social", en Boletín Cientifico Sapiens Research, vol. 4, núm. 1. [Documento www] Disponible en: http://issuu.com/sapiens-research/docs/v4n1-2014_ enero-junio_bcsr/17?e=2418709/6831311 (2014c).

Recepción: 10 de octubre de 2014.

Aprobación: 7 de julio de 2015. 